\title{
Sunitinib enhances antitumor effects against chemotherapy-resistant bladder cancer through suppression of ERK1/2 phosphorylation
}

\author{
ARIO TAKEUCHI $^{1}$, MASATOSHI ETO ${ }^{2}$, MASAKI SHIOTA ${ }^{1}$, KATSUNORI TATSUGAMI ${ }^{1}$, \\ AKIRA YOKOMIZO $^{1}$, KENTARO KUROIWA ${ }^{1}$, MOMOE ITSUMI $^{1}$ and SEIJI NAITO ${ }^{1}$ \\ ${ }^{1}$ Department of Urology, Graduate School of Medical Sciences, Kyushu University, Fukuoka; ${ }^{2}$ Department of Urology, \\ Graduate School of Medical Science, Kumamoto University, Kumamoto, Japan
}

Received October 26, 2011; Accepted December 12, 2011

DOI: $10.3892 /$ ijo.2012.1368

\begin{abstract}
Bladder cancer patients who are refractory to chemotherapy have a poor prognosis. Furthermore, additional chemotherapies provide little benefit to patients who have relapsed after an initial response. Recently, it was reported that several molecular pathways are implicated in bladder carcinogenesis, including the epidermal growth factor receptor (EGFR) pathway, the vascular endothelial growth factor (VEGF) pathway and the Ras-MAPK pathway. We hypothesized that sunitinib would be effective in bladder cancer as it is an oral inhibitor of multiple receptor tyrosine kinases, including VEGF receptors, platelet derived growth factor (PDGF) receptors and stem cell factor receptor (c-KIT), and is a standard first-line treatment of advanced clear cell renal carcinoma. In the present study, the antiproliferative effects of sunitinib were clearly demonstrated in KK47, KK47/DDP20 and KK47/ADR cell lines in vitro due to the suppression of ERK1/2 phosphorylation. In a mouse model, the antitumor effects of sunitinib were again clearly seen. Also, treatment with sunitinib decreased the abundance of regulatory $\mathrm{T}$ cells (Tregs). However, cytotoxic T lymphocyte (CTL) activity was not induced sufficiently as compared with an IFN- $\alpha$-treated group. Our results suggested that sunitinib was effective in chemotherapy-resistant bladder cancer patients. On the other hand, these findings provided the rationale for combination therapy with sunitinib and immune-based cancer therapy for advanced malignancies to prevent the occurrence of rebound phenomena.
\end{abstract}

\section{Introduction}

Bladder cancer is one of the most common types of cancer in industrialized countries (1). Patients with primary metastatic

Correspondence to: Dr Masatoshi Eto, Department of Urology, Graduate School of Medical Science, Kumamoto University, 1-1-1 Honjo, Kumamoto 860-8556, Japan

E-mail: etom@kumamoto-u.ac.jp

Key words: sunitinib, anti-tumor effect, chemotherapy-resistant, bladder cancer disease and first-line cisplatin-based combination chemotherapy with methotrexate, vinblastine, adriamycin and cisplatin (MVAC) show a long-term survival of $20 \%$ (2). However, approximately $30 \%$ of patients receiving MVAC do not respond, resulting in disease progression (3). As a result, patients who are resistant to cisplatin-based combination chemotherapy have a poor prognosis. Furthermore, additional chemotherapies provide little benefit to patients who have relapsed after an initial response (2). We have been investigating a new treatment method that is used for bladder cancer patients who are resistant to cisplatin-based chemotherapy.

Bladder cancer has been reported to develop alongside complex genetic events, which involve signal transduction, cellular proliferation, angiogenesis and apoptosis. Several molecular pathways have been implicated in bladder carcinogenesis, including the epidermal growth factor receptor (EGFR) pathway, vascular endothelial growth factor (VEGF) pathway and Ras-MAPK pathway (4). RAS proteins deliver signals from cell surface receptors, passing them from protein to protein along several different pathways (5). The RAS-RAF/MEK (mitogen extracellular kinase)/ERK (extracellular signal-related kinase) pathway, a common downstream RAS signaling pathway present in all eukaryotic cells, is upregulated in approximately $30 \%$ of all human cancers (6). Sunitinib is an oral inhibitor of multiple receptor tyrosine kinases, including VEGF receptors, platelet derived growth factor (PDGF) receptors and stem cell factor receptor (c-KIT), and is the standard first-line treatment of advanced clear cell renal carcinoma (7). The inhibition of the tyrosine kinases necessary for these growth factors to interact with their respective receptors provides a potential target for various anti-cancer therapies, inhibiting angiogenesis and tumor cell proliferation (8-10). Therefore, we postulated that sunitinib might be effective in bladder cancer.

In the present study, we evaluated the therapeutic potential and molecular mechanism of sunitinib in human and murine bladder cancers or drug-resistant human bladder cancers. Our results suggest that sunitinib is effective in MVAC-resistant bladder cancer patients accompanied with the suppression of ERK1/2 phosphorylation, overexpression of which has been recently reported to have a relation to drug resisitance (11-17). In addition, treatment with sunitinib decreased the abundance 
of regulatory $\mathrm{T}$ cells (Tregs) in a mouse model. This might be one of the causes of the appearance of anti-tumor activity. However, because cytotoxic T lymphocyte (CTL) activity was not induced sufficiently, the necessity of combination therapy with sunitinib and immune-based cancer therapy to prevent reccurrence was also postulated.

\section{Materials and methods}

Animals and reagents. Female $\mathrm{C} 3 \mathrm{H} / \mathrm{HeN}\left(\mathrm{H}-2^{\mathrm{K}}\right)$ mice (6-8 weeks old) and female NCr-nu/nu mice (6-8-weeks old) were obtained from Charles River Laboratories (Yokohama, Japan). Sunitinib (Sutent) was obtained from Pfizer (New York, $\mathrm{NY}$ ). For the in vitro and in vivo studies, sunitinib was prepared as described previously (18). Mouse interferon- $\alpha$ (IFN- $\alpha$ ) was supplied by Dainippon Sumitomo Pharma Co., Ltd. (Osaka, Japan). IFN- $\alpha$ was prepared as described previously (19).

Tumor cell line. KK47 cell lines, established from human transitional cell carcinoma of the urinary bladder, were used as parental cell lines. KK47 cell lines were cultured as described previously (20). Cisplatin-resistant KK47/DDP20 cells and adriamycin-resistant KK47/ADR cells derived from KK47 cell lines were cultured as described previously (20). MBT-2 cells, derived from a carcinogen-induced bladder tumor in a $\mathrm{C} 3 \mathrm{H}$ mouse, were cultured as described previously $(21,22)$.

Antibodies and reagents. Fluorescein isothiocyanate (FITC)conjugated anti-CD44 mAb (IM7), anti-Foxp3 mAb (FJK-16s) and anti-CD3 $\mathrm{mAb}$ (145-2C11), phycoerythrin (PE)-conjugated anti-CD25 mAb (PC61.5), anti-NK (DX5) $\mathrm{mAb}$ and anti-CD8 $\mathrm{mAb}$ (53-6.7) and PerCP-Cy5.5-conjugated anti-CD4 $\mathrm{mAb}$ (H129.19) were purchased from BD Pharmingen (San Diego, CA). Live lymphocytes were gated by forward and side scattering. The data were analyzed with CellQuest software (BD Biosciences). Antibodies against ERK1/2 (Thr202/Tyr204) were purchased from Santa Cruz Biotechnology (Santa Cruz, CA). Anti- $\beta$ actin antibody was purchased from Sigma (St. Louis, MO).

Cytotoxicity analysis. Cytotoxicity analysis was performed as previously described $(23,24)$. Briefly, KK47, KK47/ADR and KK47/DPP20 cells $\left(2.5 \times 10^{3}\right)$ were seeded into 96-well plates. The following day the indicated concentrations of mevastatin, simvastatin, and dihydrotestosterone (DHT) were added. After $72 \mathrm{~h}$, the surviving cells were stained using the Alamar Blue assay (TREK Diagnostic Systems, Cleveland, OH) for $180 \mathrm{~min}$ at $37^{\circ} \mathrm{C}$. Absorbance in each well was measured using a plate reader (ARVO ${ }^{\mathrm{TM}} \mathrm{MX}$; Perkin-Elmer, Inc., Waltham, MA).

Western blotting. Whole-cell extracts were prepared as previously described $(23,25,26)$. Protein concentrations were determined using a protein assay kit (Bio-Rad, Hercules, CA), based on the Bradford method. Whole cell extracts $(30 \mathrm{mg}$ ) were separated by sodium dodecyl sulfate-polyacrylamide gel electrophoresis (SDS-PAGE) and transferred to polyvinylidene difluoride microporous membranes (GE Healthcare Bio-Science, Piscataway, NJ) using a semi-dry blotter. Blotted membranes were incubated for $1 \mathrm{~h}$ at room temperature with primary antibody. Membranes were then incubated for $40 \mathrm{~min}$ at room temperature with peroxidase- conjugated secondary antibody. Bound antibody was visualized using an ECL kit (GE Healthcare Bio-Science) and membranes were exposed to high performance chemiluminescence film (GE Healthcare Bio-Science).

Intracellular FACS. Lymphocytes were harvested and surface stained in buffer containing PE-conjugated anti-CD25 mAb and PerCP-Cy5.5 anti-CD4 mAb. Cells were then subjected to intracellular staining using a BD fixation and permeabilization system (BD Biosciences) according to the manufacturer's instructions. The cells were fixed and permeabilized by incubation for $20 \mathrm{~min}$ at $4^{\circ} \mathrm{C}$ with $100 \mu \mathrm{l}$ of BD Cytofix/Cytoperm solution. After washing with BD perm-wash buffer, cells were stained with FITC-conjugated anti-Foxp3 mAb (FJK-16s). After intracellular staining, cellular fluorescence was analyzed using a FACSCalibur flow cytometer (BD Biosciences, San Jose, CA).

Challenge with tumor cells. MBT-2 cells $\left(5 \times 10^{6}\right)$ were inoculated subcutaneously (s.c.) into the shaved lateral flanks of mice. The mice were each challenged intragastrically (i.g.) with a suspension of $100 \mathrm{ml}$ sunitinib $(40 \mathrm{mg} / \mathrm{kg}$ ) or phosphate buffered saline (PBS) from 4 days after tumor inoculation. According to the human 4 weeks on, 2 weeks off schedule, mice were treated by sunitinib or PBS for 4 weeks before sacrificed. Sizes of primary tumors were determined every 2 or 3 days using calipers. Tumor volume was calculated using the formula $\mathrm{V}=\left(\mathrm{A}_{\mathrm{X} \mathrm{B}} \mathrm{B}^{2}\right) / 2$, where $\mathrm{V}$ is the volume $\left(\mathrm{mm}^{3}\right)$, $A$ is the long diameter $(\mathrm{mm})$ and $B$ is the short diameter $(\mathrm{mm})$.

CTL activity in the spleen. CTL activity was performed as described previously (19). Briefly, for CTL activity, splenocytes were taken from mice s.c. inoculated with MBT-2 cells on day 14 after sunitinib or PBS treatment. Subsequently, $5 \times 10^{6}$ cells $/ \mathrm{ml}$ splenocytes were cultured for 5 days with $5 \times 10^{5}$ cells $/ \mathrm{ml}$ mitomycin C (MMC)-treated MBT-2 cells in the presence of $25 \mathrm{ng} /$ $\mathrm{ml}$ IL-2 (Takeda Chemical Industries, Tokyo, Japan) diluted in $2 \mathrm{ml}$ of complete culture medium. Viable lymphocytes were separated by Percoll (Amersham Biosciences, Piscataway, $\mathrm{NJ}$ ) density gradient centrifugation and the effector cells were incubated at the indicated E:T ratio with $1 \times 10^{4}{ }^{51} \mathrm{Cr}$-labeled MBT-2 cells for $4 \mathrm{~h}$. Cytotoxicity was calculated as follows: (experimental release - spontaneous release) / (total release spontaneous release) x 100 .

Statistical analysis. Statistical significance of the data was determined using an unpaired two-tailed Student's t-test. $\mathrm{P}<0.05$ was taken as the level of significance. Analysis was carried out using Stat-View 5.0 software (Abacus Concepts, Berkeley, CA).

\section{Results}

Anti-proliferative effects of sunitinib in three bladder cancer cells lines. The inhibitory effect of statins on cell growth was examined as shown in Fig. 1. Sunitinib suppressed cell growth in KK47, KK47/DDP20 cisplatin-resistant bladder cancer cells and in KK47/ADR adriamycin-resistant bladder cancer cells in a dose-dependent manner. These data suggest that the inhibitory effect of sunitinib is effective in chemotherapy-resistant bladder cancer cells. 
A

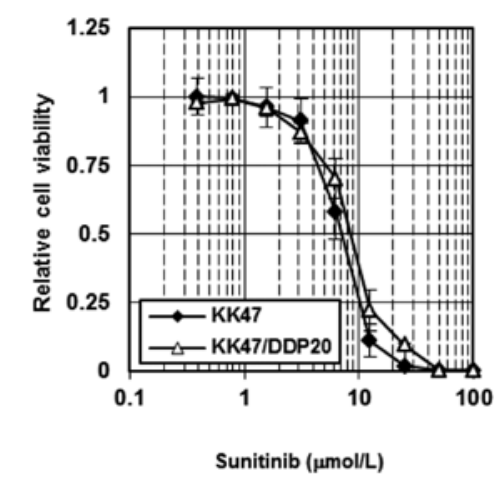

B

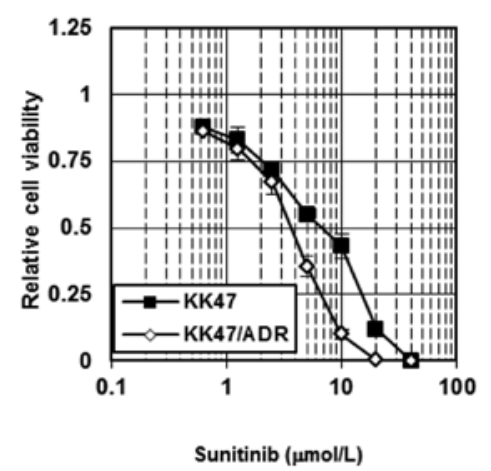

Figure 1. Growth inhibitory effect of sunitinib on bladder cancer cell lines. KK47, KK47/DPP20 and KK47/ADR cells (A) were seeded into 96-well plates. The following day, various concentrations of sunitinib were added. After $72 \mathrm{~h}$, cell survival rates were evaluated using cytotoxicity analyses. Cell survival in the absence of sunitinib was set as 1 . All values are representative of at least three independent experiments. Data represent the mean value of KK47, KK47/DPP20 and KK47/ADR cells subjected to cytotoxicity analyses.

A

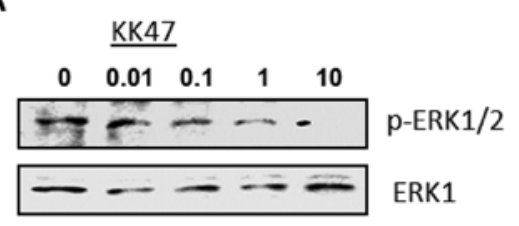

B

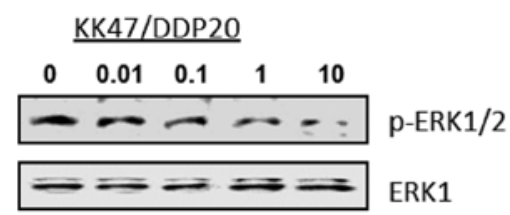

C

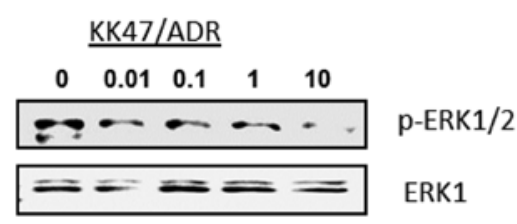

Figure 2. Downregulation of ERK1/2 phosphorylation by sunitinib. Sunitinib was added to media containing, KK47, KK47/DPP20 and KK47/ADR cells at the concentrations and durations indicated. Whole-cell extracts were subjected to SDS-PAGE. Western blotting was performed using the antibodies indicated.
A

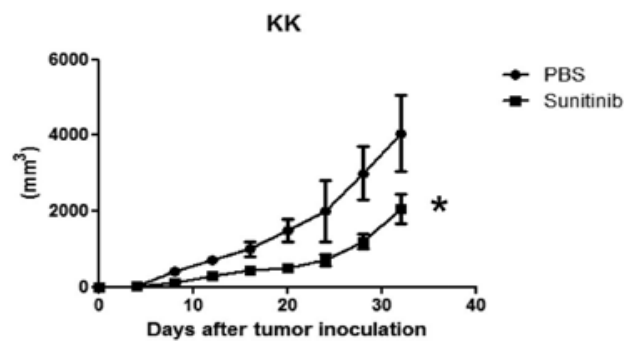

B

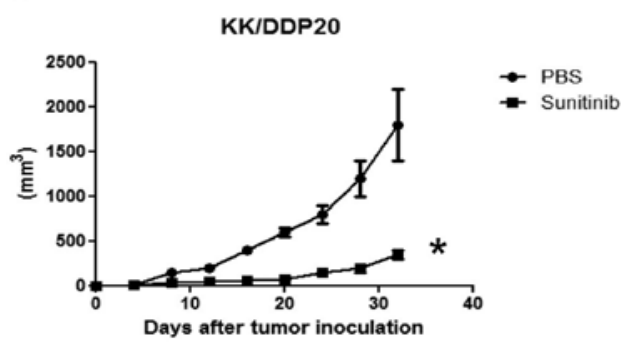

C

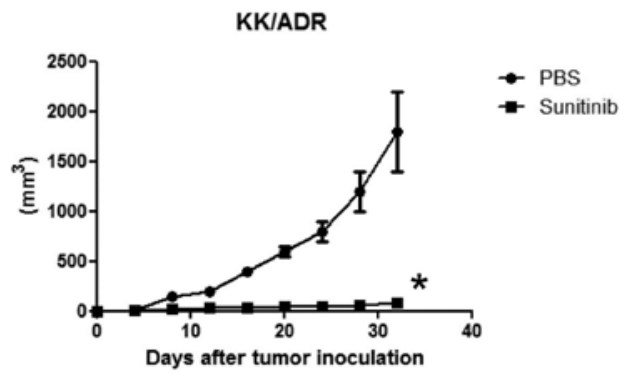

Figure 3. Antitumor effects of sunitinib in the nude mouse model. KK47 (A), KK47/DDP20 (B) or KK47/ADR (C) cells $\left(5.0 \times 10^{7}\right)$ were inoculated subcutaneously (s.c.) into the shaved lateral flanks of mice. After 4 days, 20 mice were randomly divided into two groups. These groups were treated with either sunitinib (40 mg/kg) or PBS. Sunitinib or PBS, administered orally, once a day, for 28 days. Primary tumor size was determined every 2 or 3 days using calipers. ${ }^{*} \mathrm{P}<0.05$ compared with PBS group. Each group consisted of five mice. Points, mean; bars, SD

Western blotting for ERK1/2. ERK 1/2 has been reported to be involved in drug resistance (11-17). As shown in Fig. 2, phosphorylation of ERK1/2 was downregulated in a time- and dose-dependent manner in KK47, KK47/DDP20 and KK47/ ADR cells following the addition of sunitinib.

Antitumor effects of sunitinib in the nude mouse model. Athymic nude mice were s.c. implanted with KK47, KK47/ DDP20 or KK47/ADR tumors ( $1 \times 10^{7}$ cells/body) on day 0 and treatment with sunitinib $(40 \mathrm{mg} / \mathrm{kg})$ was initiated on day 4 when tumors were established. Daily treatment with sunitinib produced a more efficient inhibition of tumor growth than PBS in all three tumor cell types (Fig. 3). Namely, the anti-tumor effects of sunitinib were observed in normal bladder cancer cells, cisplatin-resistant bladder cancer cells and adriamycinresistant bladder cancer cells.

Antitumor effects of sunitinib in the $\mathrm{C} 3 \mathrm{H} / \mathrm{HeN}$ mouse model. Treatment with sunitinib (40 mg/kg) was initiated on day 4 


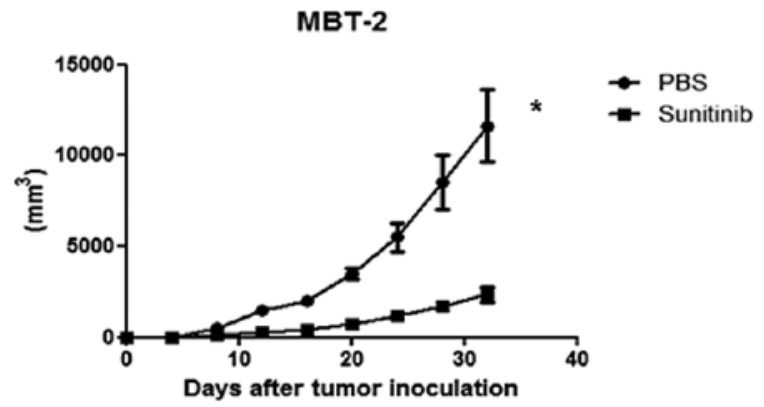

Figure 4. Anti-tumor effect of sunitinib in a mouse model. MBT-2 cells were inoculated subcutaneously (s.c.) into the shaved lateral flanks of mice. After 4 days, 20 mice were randomly divided into two groups. These groups were treated with either sunitinib $(40 \mathrm{mg} / \mathrm{kg}$ ) or PBS. Sunitinib or PBS, administered orally, once a day, for 28 days. Primary tumor size was determined every 2 or 3 days using calipers. ${ }^{*} \mathrm{P}<0.05$ compared with PBS group. Each group consisted of five mice. Points, mean; bars, SD.

A The number of splenocytes

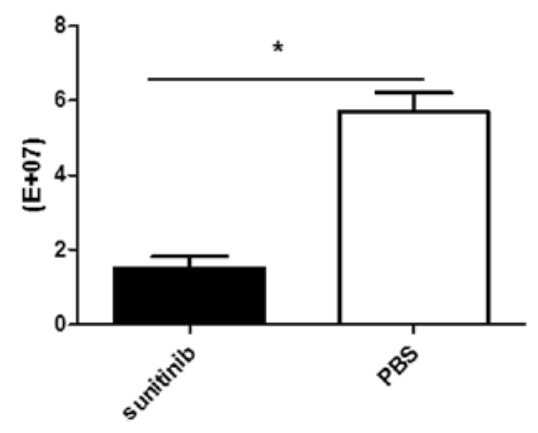

C The percentage of CD8+ T cells

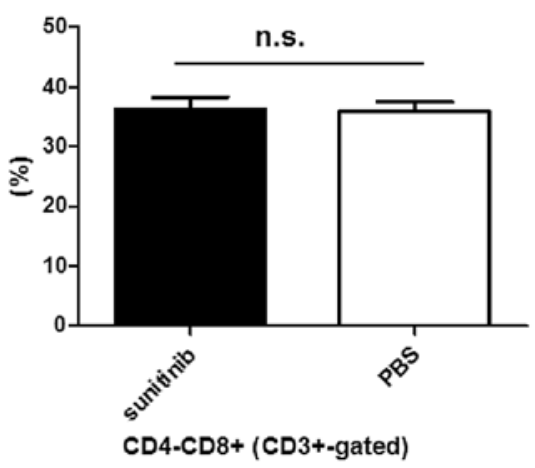

E The percentage of regulatory $T$ cells

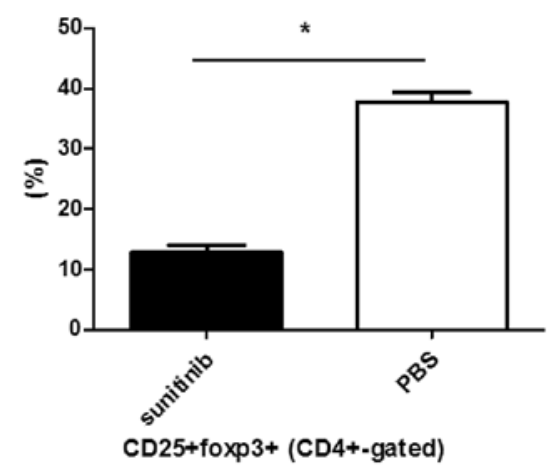

after MBT-2 $\left(5.0 \times 10^{6}\right)$. Tumor growth was also significantly suppressed in sunitinib treated mice as compared with those treated with PBS (Fig. 4).

Effect of sunitinib on T cells and generation of tumor-specific CTL activity in splenocytes of tumor-bearing $\mathrm{C} 3 \mathrm{H} / \mathrm{HeN}$ mice. We analyzed the effector cells for anti-tumor activity in the two groups described above with flow cytometry on day 21 after s.c. inoculation with MBT-2 cells. There were no significant differences in the proportions of $\mathrm{CD}^{-} \mathrm{NK} 1.1^{+}$cells (NK cells) (Fig. 5B), CD8 ${ }^{+} \mathrm{T}$ cells (Fig. 5C), and CD4 ${ }^{+} \mathrm{T}$ cells (Fig. 5D). However, significant decrease in the number of splenocytes and the proportion of $\mathrm{CD} 4^{+} \mathrm{CD} 25^{+}$Foxp $^{+} \mathrm{T}$ cells (regulatory $\mathrm{T}$ cells) were observed among the two groups (Fig. 5A and E).

Next, to assess the establishment of acquired immunity against MBT-2, CTL activity against MBT-2 was examined in

B

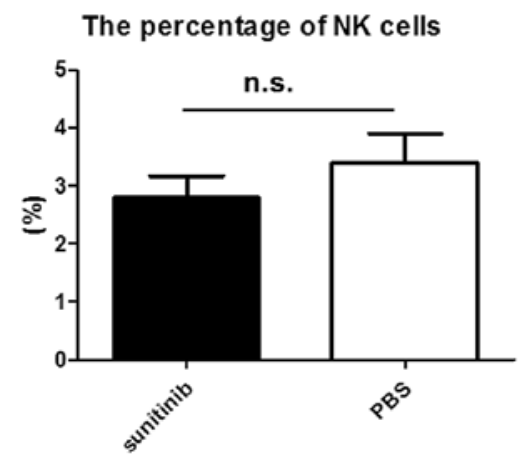

NK1.1 + CD3- (lymphocyte-gated)

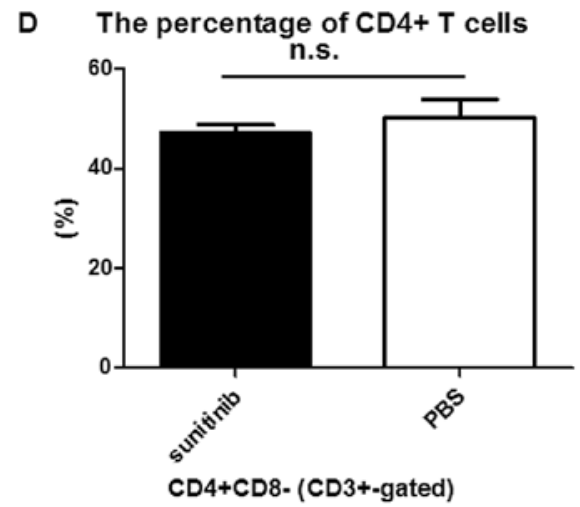

Figure 5. Effects of sunitinib on the proportion of lymphocytes and the number of splenocytes. The number of splenocytes were examined by a counting chamber (A) and a FACSCalibur flow cytometer at 32 days after tumor inoculation. $\mathrm{NK} 1.1^{+} \mathrm{CD} 3{ }^{-}, \mathrm{CD} 4^{+} \mathrm{CD} 8, \mathrm{CD}^{-} \mathrm{CD}^{+}$, and $\mathrm{CD} 25^{+}$foxp3 ${ }^{+}$cells demonstrate NK cells (B), CD8 T cells (C), CD4 T cells (D), and reguratory $\mathrm{T}$ cells (E) respectively. (E) Representative FACS patterns of $\mathrm{CD} 4^{+} \mathrm{CD} 25^{+}$ Foxp $3^{+}$after either sunitinib or PBS treatment (ns, not specific compared with the cyclophosphamide-treated group; ${ }^{*} \mathrm{P}<0.05$ as compared with the PBStreated group). 


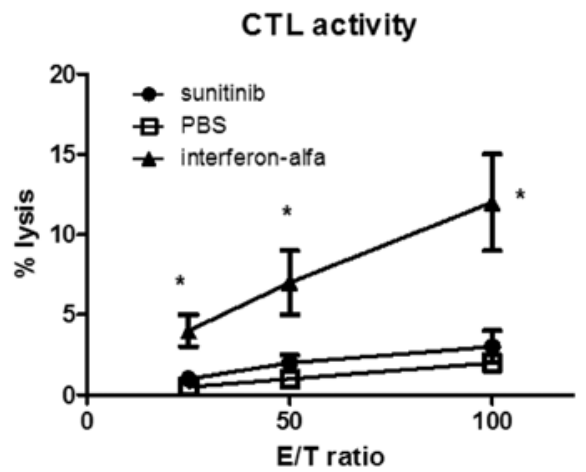

Figure 6. Effect of sunitinib on generation of tumor-specific CTL activity in spleen cells from tumor-bearing $\mathrm{C} 3 \mathrm{H} / \mathrm{HeN}$ mice. Spleen cells were removed 14 days after tumor inoculation, and used for the assay of CTL activity. ${ }^{*} \mathrm{P}<0.05$ compared with PBS and sunitinib groups. E:T ratio, effector cells:target cells ratio; CTL, cytotoxic T lymphocyte. Each group consisted of five mice. Points, mean; bars, SD.

the splenocytes of tumor-bearing $\mathrm{C} 3 \mathrm{H} / \mathrm{HeN}$ mice. In this study we used IFN- $\alpha$ as a positive control. In fact, CTL activity was easily induced in the mice treated with INF- $\alpha$. The mice treated with IFN- $\alpha$ were each challenged intraperitoneally (i.p.) with a suspension of $100 \mathrm{ml} \mathrm{IFN- \alpha} \mathrm{(5000} \mathrm{U/body)} 4$ days after tumor inoculation. According to the sunitinib-treated mouse schedule, mice were treated once a day prior to sacrifice. As a result, IFN- $\alpha$ induced higher level of CTL activity as compared with control, however sunitinib did not induce significant CTL activity as compared with control (Fig. 6).

\section{Discussion}

Currently, metastatic bladder cancer is best managed by chemotherapy, although several multi-agent chemotherapy regimens have been used, no combination has demonstrated an incremental benefit to the MVAC regimen introduced $>20$ years ago (27). However, MVAC has been shown to be associated with significant toxicities, including leukopenia, thrombocytopenia, grade $3 / 4$ mucositis, nausea, vomiting and sepsis-related death in $2-3 \%$ of cases (3). While, gemcitabine and cisplatin (GC) might be less toxic than MVAC as demonstrated by lower episodes of toxic death (1 versus 3\%) and fewer occurrences of neutropenia, fever, sepsis, mucositis and alopecia in a recent randomized study, GC did not improve the overall survival achieved with MVAC (28). About $30 \%$ of patients receiving MVAC do not respond, resulting in disease progression (3). Identification of new treatment alternatives for patients with resistant disease remains a priority and novel molecular targets are being explored.

Recently, bladder cancer has been reported to develop alongside complex genetic events, which involve signal transduction, cellular proliferation, angiogenesis and apoptosis. Several molecular pathways have been implicated in bladder carcinogenesis, including the EGFR pathway, VEGF pathway and Ras-MAPK pathway (4). Therefore, we investigated the use of sunitinib. Sunitinib is an oral inhibitor with antiangiogenic and antitumor activities and inhibits multiple receptor tyrosine kinases, including VEGF receptors, PDGF receptors and c-KIT, which are involved in angiogenesis (7). The antitumor activity of sunitinib has been shown in preclinical and clinical studies $(7,18)$. Tumor regression has been shown in different xenograft models of colon cancer, breast cancer, non-small cell lung cancer, melanoma, glioblastoma and renal carcinoma (18,29-33). Sunitinib clearly inhibited cell proliferation in vitro and tumor growth in vivo in the present study (Figs. 1, 3 and 4) Moreover, we found cancer cell proliferation to be inhibited accompanied with suppression of ERK1/2 phosphorylation as shown in Fig. 2. ERK1/2 is a member of the classical MAPK cascade, which is involved in the regulation of cell growth and survival, and its upregulation leads to abnormal cell proliferation and tumorigenicity $(4,6,34,35)$.

Recently, ERK 1/2 has been reported to be involved in drug resistance (11-17). The ERK1/2 signaling pathway could be an important therapeutic target for overcoming chemoresistance. Interestingly, in the present study sunitinib was effective in cisplatin- and adriamycin-resistant bladder cancer, as well as a cisplatin- and adriamycin-sensitive bladder cancer, accompanied with suppression of the ERK1/2 phosphorylation (Figs. 1 and 2). The results that sunitinib treatment inhibits the proliferation of drug-resistant ERK1/2-overexpressing bladder cancer cells are promising for the future treatment of chemotherapy-resistant bladder cancer.

Although anti-tumor immunity is usually suppressed in tumor-bearing mice, due to the presence of Tregs and suppressive cytokines, such as TGF- $\beta$ and IL-10, IFN- $\alpha$ is reported to induce tumor antigen peptide-specific CTL activity in patients with chronic myelogenous leukemia $(36,37)$. Our previous study also reported the induction of CTL activity against RENCAs (murine renal cell carcinoma cells) in the spleen cells of mice treated with IFN- $\alpha$ (19). An important finding of the present study was that CTL activity against MBT-2 was not induced in the spleen cells of mice treated with sunitinib, compared with mice treated with IFN- $\alpha$ (Fig. 6). This fact might be connected with the so-called 'rebound phenomenon', which is a rapid cancer progression soon after the treatment with molecular target drugs is stopped (19). Next, we investigated if sunitinib treatment also affected the percentage of Treg in tumor-bearing mice, using flow cytometry. Sunitinib treatment significantly decreased the percentage of $\mathrm{CD} 4{ }^{+} \mathrm{CD} 25^{+} \mathrm{Foxp} 3^{+}$Tregs in tumorbearing mice, whereas it did not change the percentage of other lymphocytes (Fig. 5). The correlation of molecular targeting therapy with Tregs has been recently reported. Sunitinib is reported to reduce Tregs in peripheral blood mononuclear cells of patients with MRCC and mice with colon cancer (29). Our results are compatible with these findings. The current study has implications for new therapeutic approaches based on the manipulation of Tregs. Tregs might be an important target in future therapies against cancer. It has been reported that Tregs produce immunosuppressive cytokines, such as IL-10 and TGF- $\beta$ (38-40). It is well known that Tregs are responsible for inducing and maintaining peripheral tolerance and the negative regulation of immunity $(21,41)$. The depletion of Tregs would eliminate immune-suppression mediated by Tregs leading to enhanced T-cell activity. This fact might reflect the result that higher CTL activitity treated with sunitinib was induced as compared with that treated with PBS, although significant difference was not observed (Fig. 6). However, sunitinib was also reported to be associated with significant declines in total number of leucocyte (-48\%), neutrophil (-62\%), CD3 total T cell $(-31 \%)$ and CD4 counts $(-32 \%)(42)$. In fact, the number of 
splenocytes was significantly decreased by sunitinib treatment in the present study (Fig. 5A). We thought suninitib was efficient drug for various cancers and associated with inhibition of specific lymphocyte subsets which had implications for the immunological control. We should consider these methods in the future.

In conclusion, our results show that sunitinib has an antitumor effect in murine models of bladder cancer when used as a single agent. In addition, sunitinib also exhibits a potent effect on a chemotherapy-resistant bladder cancer. Since this patient group has few options that lead to prolonged survival, sunitinib may be a promising novel therapeutic alternative in chemotherapy-refractory bladder cancer patients. On the other hand, our data suggest that sunitinib might be used to enhance the therapeutic efficacy of existing immune-based therapies for metastatic cancer patients.

\section{References}

1. Jemal A, Siegel R, Ward E, et al: Cancer statistics, 2008. CA Cancer J Clin 58: 71-96, 2008.

2. Gallagher DJ and Milowsky MI: Bladder cancer. Curr Treat Options Oncol 10: 205-215, 2009.

3. Sternberg CN, Yagoda A, Scher HI, et al: M-VAC (methotrexate, vinblastine, doxorubicin and cisplatin) for advanced transitional cell carcinoma of the urothelium. J Urol 139: 461-469, 1988.

4. Zachos I, Konstantinopoulos PA, Tzortzis V, et al: Systemic therapy of metastatic bladder cancer in the molecular era: current status and future promise. Expert Opin Investig Drugs 19: 875-887, 2010.

5. Sridhar SS, Hedley D and Siu LL: Raf kinase as a target for anticancer therapeutics. Mol Cancer Ther 4: 677-685, 2005.

6. Gollob JA, Wilhelm S, Carter C, et al: Role of Raf kinase in cancer: therapeutic potential of targeting the Raf/MEK/ERK signal transduction pathway. Semin Oncol 33: 392-406, 2006.

7. Mendel DB, Laird AD, Xin X, et al: In vivo antitumor activity of SU11248, a novel tyrosine kinase inhibitor targeting vascular endothelial growth factor and platelet-derived growth factor receptors: determination of a pharmacokinetic/pharmacodynamic relationship. Clin Cancer Res 9: 327-337, 2003.

8. Cohen HT and McGovern FJ: Renal-cell carcinoma. N Engl J Med 353: 2477-2490, 2005.

9. Oka H, Chatani Y, Hoshino R, et al: Constitutive activation of mitogen-activated protein (MAP) kinases in human renal cell carcinoma. Cancer Res 55: 4182-4187, 1995.

10. Van Spronsen DJ, De Weijer KJ, Mulders PF, et al: Novel treatment strategies in clear-cell metastatic renal cell carcinoma. Anticancer Drugs 16: 709-717, 2005.

11. Jeong EK, Lee SY, Jeon HM, et al: Role of extracellular signalregulated kinase (ERK)1/2 in multicellular resistance to docetaxel in MCF-7 cells. Int J Oncol 37: 655-661, 2010.

12. Peng DJ, Wang J, Zhou JY, et al: Role of the Akt/mTOR survival pathway in cisplatin resistance in ovarian cancer cells. Biochem Biophys Res Commun 394: 600-605, 2010.

13. Abedini MR, Muller EJ, Bergeron R, et al: Akt promotes chemoresistance in human ovarian cancer cells by modulating cisplatin-induced, p53-dependent ubiquitination of FLICE-like inhibitory protein. Oncogene 29: 11-25, 2010.

14. Mirmohammadsadegh A, Mota R, Gustrau A, et al: ERK1/2 is highly phosphorylated in melanoma metastases and protects melanoma cells from cisplatin-mediated apoptosis. J Invest Dermatol 127: 2207-2215, 2007.

15. Grandage VL, Gale RE, Linch DC, et al: PI3-kinase/Akt is constitutively active in primary acute myeloid leukaemia cells and regulates survival and chemoresistance via NF-kappaB, Mapkinase and p53 pathways. Leukemia 19: 586-594, 2005.

16. Datta SR, Brunet A and Greenberg ME: Cellular survival: a play in three Akts. Genes Dev 13: 2905-2927, 1999.

17. Song G, Ouyang $\mathrm{G}$ and Bao S: The activation of Akt/PKB signaling pathway and cell survival. J Cell Mol Med 9: 59-71, 2005.

18. Maita S, Yuasa T, Tsuchiya N, et al: Antitumor effect of sunitinib against skeletal metastatic renal cell carcinoma through inhibition of osteoclast function. Int J Cancer 130: 677-684, 2011.
19. Takeuchi A,Eto M, Tatsugami K, et al: Mechanism of synergistic antitumor effect of sorafenib and interferon-alpha on treatment of renal cell carcinoma. J Urol 184: 2549-2556, 2010.

20. Kotoh S, Naito S, Yokomizo A, et al: Increased expression of DNA topoisomerase I gene and collateral sensitivity to camptothecin in human cisplatin-resistant bladder cancer cells. Cancer Res 54: 3248-3252, 1994.

21. Takeuchi A,Eto M, Yamada $\mathrm{H}$, et al: A reduction of recipient regulatory $\mathrm{T}$ cells by cyclophosphamide contributes to an anti-tumor effect of nonmyeloablative allogeneic stem cell transplantation in mice. Int J Cancer Feb 23. doi: 10.1002/ijc.26009, 2011.

22. Kamiryo Y, Eto M, Yamada H, et al: Donor CD4 T cells are critical in allogeneic stem cell transplantation against murine solid tumor. Cancer Res 69: 5151-5158, 2009.

23. Shiota M, Yokomizo A, Tada Y, et al: Castration resistance of prostate cancer cells caused by castration-induced oxidative stress through Twist1 and androgen receptor overexpression. Oncogene 29: 237-250, 2010.

24. Shiota M, Izumi H, Tanimoto A, et al: Programmed cell death protein 4 down-regulates Y-box binding protein-1 expression via a direct interaction with Twistl to suppress cancer cell growth. Cancer Res 69: 3148-3156, 2009.

25. Shiota M, Yokomizo A, Tada Y, et al: Peroxisome proliferatoractivated receptor gamma coactivator-1alpha interacts with the androgen receptor (AR) and promotes prostate cancer cell growth by activating the AR. Mol Endocrinol 24: 114-127, 2010.

26. Shiota M, Yokomizo A, Masubuchi D, et al: Tip60 promotes prostate cancer cell proliferation by translocation of androgen receptor into the nucleus. Prostate 70: 540-554, 2010.

27. Wilhelm S, Carter C, Lynch M, et al: Discovery and development of sorafenib: a multikinase inhibitor for treating cancer. Nat Rev Drug Discov 5: 835-844, 2006.

28. El Karak F and Flechon A: Gemcitabine in bladder cancer. Expert Opin Pharmacother 8: 3251-3256, 2007.

29. Ozao-Choy J, Ma G, Kao J, et al: The novel role of tyrosine kinase inhibitor in the reversal of immune suppression and modulation of tumor microenvironment for immune-based cancer therapies. Cancer Res 69: 2514-2522, 2009.

30. Gandhi L, McNamara KL, Li D, et al: Sunitinib prolongs survival in genetically engineered mouse models of multistep lung carcinogenesis. Cancer Prev Res (Phila) 2: 330-337, 2009.

31. Kujawski M, Zhang C, Herrmann A, et al: Targeting STAT3 in adoptively transferred $\mathrm{T}$ cells promotes their in vivo expansion and antitumor effects. Cancer Res 70: 9599-9610, 2010.

32. Young E, Miele L, Tucker KB, et al: SU11248, a selective tyrosine kinases inhibitor suppresses breast tumor angiogenesis and growth via targeting both tumor vasculature and breast cancer cells. Cancer Biol Ther 10: 703-711, 2010.

33. Schueneman AJ, Himmelfarb E, Geng L, et al: SU11248 maintenance therapy prevents tumor regrowth after fractionated irradiation of murine tumor models. Cancer Res 63: 4009-4016, 2003.

34. Chen Z, Gibson TB, Robinson F, et al: MAP kinases. Chem Rev 101: 2449-2476, 2001.

35. Zeng P, Wagoner HA, Pescovitz $\mathrm{OH}$, et al: RNA interference (RNAi) for extracellular signal-regulated kinase 1 (ERK1) alone is sufficient to suppress cell viability in ovarian cancer cells. Cancer Biol Ther 4: 961-967, 2005.

36. Frumento G, Piazza T, Di Carlo E, et al: Targeting tumor-related immunosuppression for cancer immunotherapy. Endocr Metab Immune Disord Drug Targets 6: 233-237, 2006.

37. Molldrem JJ, Lee PP, Wang C, et al: Evidence that specific T lymphocytes may participate in the elimination of chronic myelogenous leukemia. Nat Med 6: 1018-1023, 2000.

38. Somasundaram R, Jacob L, Swoboda R, et al: Inhibition of cytolytic $\mathrm{T}$ lymphocyte proliferation by autologous $\mathrm{CD} 4^{+} / \mathrm{CD} 25^{+}$ regulatory $\mathrm{T}$ cells in a colorectal carcinoma patient is mediated by transforming growth factor-beta. Cancer Res 62: 5267-5272, 2002.

39. Sakaguchi S: Regulatory T cells: key controllers of immunologic self-tolerance. Cell 101: 455-458, 2000.

40. Liyanage UK, Moore TT, Joo HG, et al: Prevalence of regulatory $\mathrm{T}$ cells is increased in peripheral blood and tumor microenvironment of patients with pancreas or breast adenocarcinoma. $\mathbf{J}$ Immunol 169: 2756-2761, 2002.

41. Von Boehmer H: Mechanisms of suppression by suppressor $\mathrm{T}$ cells. Nat Immunol 6: 338-344, 2005.

42. Powles T, Chowdhury S, Bower M, et al: The effect of sunitinib on immune subsets in metastatic clear cell renal cancer. Urol Int 86: 53-59, 2011. 